\title{
Event-Based Stabilization over Networks with Transmission Delays
}

\author{
Xiangyu Meng and Tongwen Chen \\ Department of Electrical and Computer Engineering, University of Alberta, Edmonton, AB, Canada T6G 2V4 \\ Correspondence should be addressed to Xiangyu Meng, xmeng2@ece.ualberta.ca
}

Received 29 November 2011; Accepted 19 February 2012

Academic Editor: Peter X. Liu

Copyright ( $) 2012$ X. Meng and T. Chen. This is an open access article distributed under the Creative Commons Attribution License, which permits unrestricted use, distribution, and reproduction in any medium, provided the original work is properly cited.

This paper investigates asymptotic stabilization for linear systems over networks based on event-driven communication. A new communication logic is proposed to reduce the feedback effort, which has some advantages over traditional ones with continuous feedback. Considering the effect of time-varying transmission delays, the criteria for the design of both the feedback gain and the event-triggering mechanism are derived to guarantee the stability and performance requirements. Finally, the proposed techniques are illustrated by an inverted pendulum system and a numerical example.

\section{Introduction}

Traditional control theory is built on the idea of perfect information flow from the sensor to the controller and from the controller to the actuator, that is, there is no delay and the transmitted signals are equal to received signals. However, this is not true for control loop closed over networks, where the actuators, sensors, and controllers are distributed in a wide geographical area, operating via some communication networks, such as DeviceNet, Ethernet, and FireWire, to name a few [1]. Because of the network uncertainties, data packets can be delayed, dropped, or reordered which make closed-loop control very difficult. Therefore, control over networks appears and has been drawing more and more attention in recent years from researchers working in the areas of systems and control [2-5]. A typical feature in the literature lies in the periodic execution of the control task due to the ease of analysis and design. However, the time synchronization problem presents a challenge in digital control applications when dealing with multiple sampling rates and systems with distributed computing devices; sampling jitter, time-varying delays, and coding errors introduced by networked distributed systems may degrade the performance or even cause closed-loop instability. On the other hand, periodic sampling only considers the system dynamics at every sampling instance triggered by a clock, and it does not take into account the constraints of both computer resources and communication bandwidth. Hence, the communication resources usage in this control scheme is inefficient.

To relax the periodicity assumption, event triggering tech-niques are proposed. Various terms are used to express event-based sampling strategy: the level crossing sampling [6], the magnitude-driven sampling, and, sometimes, sampling in the amplitude domain, Lebsegue sampling [7]. In the sensor network community, the magnitude-driven or level crossing sampling is known as send-on-delta [8] or deadbands [9]. By contrast, event-based communication mechanisms use resources more efficiently by invoking operation only when a specific event occurs in the system, which guarantees relatively little communication effort. Due to easy implementation, event-based control mechanisms have been used in industry for some time, ranging in sectors from oil and gas, power and utility, to manufacturing. Unfortunately there is little methodology available for event-based control at the beginning. This could be explained as the mathematical difficulties since event-based control integrates discrete logic functions with continuously evolving system dynamics. However, most existing control design methods focus only on dynamic systems, while ignoring logic constraints. Early results on discontinuous systems and impulsive control were 
used to solve problems concerning event-based control. Event-based control can also be viewed as a special case of hybrid systems from the viewpoint of the continuous variables and discrete transition associated with events. Recently, a few fundamental results have been reported for event-based control [10-18] and estimation [19]. Overall, the research of event-based control is still in its infancy, and the results obtained are still very limited, contrasting to its wider applications in practical control problems. The various benefits of event-based control necessitate overcoming the difficulties in the analysis and design of this type of control strategies.

In this paper, further results on event-based control recently dealt with in [10] are presented, where an event generation condition based on the control error is proposed rather than the state error considered in [10]. This is achieved by adopting the topology that the sensor, controller, and event detector reside on the same node in the network. The event detector contains sophisticated logic devices to trigger an event when the control error norm reaches a certain proportion of the state norm, and then send the current control signal to the actuator node. The effect of time-varying network transmission delays is considered instead of a constant computational delay as in [10]. The defined events guarantee that the controller designed can stabilize the event-based control system. The relationships between the parameters of the event detector, the upper bound of transmission delays, and the feedback gain are also established. Moreover, the feasibility of this eventbased scheme is verified by estimating the lower bound of the difference between two consecutive event times. Two simulation examples are presented to illustrate the proposed approach.

The rest of this paper is organized as follows. Section 2 presents the event-triggered problem with consideration of time-varying transmission delays. Based on a control error event-triggered scheme, design for both the controller and the parameter of the event detector for event-based control systems is proposed in Section 3. Two simulation examples are given in Section 4 to demonstrate the advantage of the event-triggered algorithm. Finally, Section 5 concludes the paper.

\section{Problem Statement}

Consider the following continuous-time linear system described by

$$
\dot{x}(t)=A x(t)+B u(t)
$$

where $x(t) \in \mathbb{R}^{n}$ and $u(t) \in \mathbb{R}^{m}$ denote the system state vector, and control input, respectively. We make the assumption that all state variables are measurable. The parameter matrices $A$ and $B$ are known with appropriate dimensions.

Different from traditional control systems that the interconnection between the plant and the actuator is transparent, the actuator considered is connected to the system in (1) through a communication link. In this case, the usual

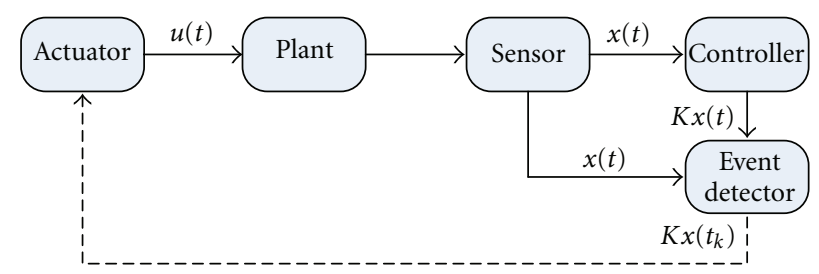

Figure 1: Event-based control loop.

assumption that the transmitted signals are equal to received signals is no longer applicable. As is well known, the periodic sampling mechanism has been used implicitly for a few decades due to the ease of analysis and design. However, the communication resources may be used unnecessarily in some situations. The event-based control scheme is used in this paper to avoid the overload of the network transmission and reduce the network bandwidth usage.

The topological structure of the considered event-based control system consisting of a continuous-time linear timeinvariant system, a sensor, a continuous-time state-feedback controller, an event detector, and an actuator is shown in Figure 1. The signal is transmitted continuously along the solid lines among the actuator, the plant, and the smart event detector; whereas the communication link denoted by the dash line is only used after an event has been generated. As Figure 1 suggests, the sensor, controller, and event detector reside on the same node in the network. The sensor samples the plant continuously, then the sampled signals are sent to the controller and the event detector. The event detector determines whether the control signal will be sent out through the network by using the event condition. Under the event condition, a sequence of time instants, $t_{0}, t_{1}, t_{2}, \ldots$, is determined as the event time, where $t_{0}=0$ is the initial time. The inter-event time is defined as $t_{k+1}-t_{k}$ which corresponds to the release period given by the event detector. Throughout this paper, it is assumed that the elapsed time between the sensor measurement and the event detector decision is negligible, the data is transmitted with a single packet, and packet loss does not occur in transmission. Thus the only effect considered for network uncertainties is the transmission delays on the system. Suppose the delay $\tau_{k}$ in the network communication is time varying and $\tau_{k} \in$ $[0, \tau]$, where $\tau$ is a constant scalar representing the maximum delay. The control signal $u\left(t_{0}\right), u\left(t_{1}\right), u\left(t_{2}\right), \ldots$ will arrive at the actuator side at the instants $t_{0}+\tau_{0}, t_{1}+\tau_{1}, t_{2}+\tau_{2}, \ldots$, respectively. Moreover, the actuator will hold the control value $u\left(t_{k}\right)$ and drive the controlled plant until a new message arrives. Therefore, the output of the actuator can be expressed as

$$
u(t)=K x\left(t_{k}\right), \quad \text { for } t \in\left[t_{k}+\tau_{k}, t_{k+1}+\tau_{k+1}\right),
$$

where $K$ is a state feedback gain matrix of appropriate dimension to stabilize $A+B K$. 
Based on the above analysis, the system model under the controller in (2) with event-based communication over networks can be described by

$$
\dot{x}(t)=A x(t)+B K x\left(t_{k}\right), \quad \text { for } t \in\left[t_{k}+\tau_{k}, t_{k+1}+\tau_{k+1}\right) \text {. }
$$

The purpose of this paper is to design an event condition rendering the event-based control system in (3) asymptotically stable.

\section{Main Results}

Inspired by [10], the event detector has the following form:

$$
\left\|e_{k}(t)\right\|<\sigma\|x(t)\|
$$

where $e_{k}(t)$ is defined as the control error between the currently computed control value and the previously submitted one

$$
e_{k}(t)=K\left(x\left(t_{k}\right)-x(t)\right), \quad \text { for } t \in\left[t_{k}, t_{k+1}\right)
$$

and $\sigma$ is a positive scalar to be determined later.

Remark 1. In the literature, event generation is usually based on the difference between the current plant state and the previously submitted plant state or the difference between the plant state and the state of a reference model. The results presented in this paper are based on the assumption that the event detector is implemented with respect to control errors. This does make sense in a networked control setting where the shared resource is the transmission medium.

Remark 2. To reduce communication bus load, the computed control signal satisfying the event condition in (4) will not be sent to update the actuator. Only the one that violates the inequality in (4) will be transmitted, but the communication will not be invoked when the system is in steady state. Intuitively, decreasing the value of $\sigma$ has the effect of shrinking the average inter-event time since the ratio between the control error and the state will need less time to reach the threshold as the value of $\sigma$ decreases. Particularly, the event-triggered scheme reduces to a continuous communication case when $\sigma=0$.

Combining the definition of $e_{k}(t)$, the dynamic of the digitally implemented control system for $t \geq t_{k}+\tau_{k}$ can be described by

$$
\begin{aligned}
\dot{x}(t) & =A x(t)+B K x\left(t_{k}\right) \\
& =(A+B K) x(t)+B K\left(x\left(t_{k}\right)-x(t)\right) \\
& =(A+B K) x(t)+B e_{k}(t) .
\end{aligned}
$$

If we consider the control error as a perturbation, it is natural to apply the perturbation method [20]. The input-to-state stable Lyapunov function candidate $V(x, t)=$ $x^{T}(t) P x(t)$ with respect to control errors $e(t)$ is used to investigate the stability for the event-based control system.
The derivative of $V(x, t)$ along the trajectories of (6) is given by

$$
\begin{aligned}
\frac{\partial V(x, t)}{\partial t}= & \frac{\partial V(x, t)}{\partial x(t)}\left((A+B K) x(t)+B e_{k}(t)\right) \\
= & x^{T}(t)\left((A+B K)^{T} P+P(A+B K)\right) x(t) \\
& +x^{T}(t) P B e_{k}(t)+e_{k}^{T}(t) B^{T} P x(t) \\
= & -x^{T}(t) Q x(t)+2 x^{T}(t) P B e_{k}(t),
\end{aligned}
$$

where $Q$ is a symmetric matrix defined by

$$
(A+B K)^{T} P+P(A+B K)+Q=0 .
$$

From (7), it can be obtained that

$$
\frac{\partial V(x, t)}{\partial t} \leq-\lambda_{\min }(Q)\|x\|^{2}+2\|P B\|\|x\|\left\|e_{k}\right\| .
$$

Thus based on the Lasalle's invariance principle, a sufficient condition to guarantee the asymptotic stability is

$$
\left\|e_{k}(t)\right\|<\sigma_{1}\|x(t)\|
$$

where

$$
\sigma_{1}=\frac{\lambda_{\min }(Q)}{2\|P B\|} .
$$

The next event instant is given by

$$
t_{k+1}=\inf \left\{t>t_{k} \mid\left\|e_{k}(t)\right\| \geq \sigma\|x(t)\|\right\} .
$$

Choose $\sigma \leq \sigma_{1}$, which implies the asymptotic stability of the system in (3) for $t \in\left[t_{k}+\tau_{k}, t_{k+1}\right)$ since the inequality in (10) is satisfied.

Now consider the interval $\left[t_{k+1}, t_{k+1}+\tau_{k+1}\right)$. First look at the dynamic of $\left\|e_{k}(t)\right\| /\|x(t)\|$ :

$$
\begin{aligned}
\frac{d}{d t} \frac{\left\|e_{k}(t)\right\|}{\|x(t)\|}= & \frac{d}{d t} \frac{\left[e_{k}^{T}(t) e_{k}(t)\right]^{1 / 2}}{\left[x^{T}(t) x(t)\right]^{1 / 2}} \\
= & -\frac{e_{k}^{T}(t) K \dot{x}(t)}{\left\|e_{k}(t)\right\|\|x(t)\|}-\frac{x(t)^{T} \dot{x}(t)}{\|x(t)\|^{2}} \frac{\left\|e_{k}(t)\right\|}{\|x(t)\|} \\
\leq & \frac{\left\|e_{k}(t)\right\|\|K\|\|\dot{x}(t)\|}{\left\|e_{k}(t)\right\|\|x(t)\|}+\frac{\|x(t)\|\|\dot{x}(t)\|}{\|x(t)\|\|x(t)\|} \frac{\left\|e_{k}(t)\right\|}{\|x(t)\|} \\
= & \left(\|K\|+\frac{\left\|e_{k}(t)\right\|}{\|x(t)\|}\right) \frac{\|\dot{x}(t)\|}{\|x(t)\|} \\
\leq & \left(\|K\|+\frac{\left\|e_{k}(t)\right\|}{\|x(t)\|}\right) \\
& \times \frac{\|A+B K\|\|x(t)\|+\|B\|\left\|e_{k}(t)\right\|}{\|x(t)\|} \\
= & \|K\|\|A+B K\|+(\|K\|\|B\|+\|A+B K\|) \\
& \times \frac{\left\|e_{k}(t)\right\|}{\|x(t)\|}+\|B\|\left(\frac{\left\|e_{k}(t)\right\|}{\|x(t)\|}\right)^{2} .
\end{aligned}
$$

The comparison lemma in [20] as a tool to compute bounds on a solution without computing the solution itself 
can be used to estimate the bound on $\left\|e_{k}(t)\right\| /\|x(t)\|$. Consider the scalar differential equation

$$
\dot{\varphi}(t)=\alpha \varphi^{2}(t)+(\alpha \beta+\gamma) \varphi(t)+\beta \gamma,
$$

where

$$
\alpha=\|B\|, \quad \beta=\|K\|, \quad \gamma=\|A+B K\| .
$$

Let $\left[t_{k+1}, t_{k+1}+\tau\right)$ be the interval of existence of the solution $\varphi(t)$. Recall that (12) implies $e_{k}(t)=\sigma x(t)$ at event instant $t=t_{k+1}$ and thus $\left\|e_{k}\left(t_{k+1}\right)\right\| /\left\|x\left(t_{k+1}\right)\right\|=\sigma$. Let

$$
\sigma \leq \varphi\left(t_{k+1}\right)=\sigma_{2}<\sigma_{1} .
$$

Then $\left\|e_{k}(t)\right\| /\|x(t)\| \leq \varphi(t)$ for all $t \in\left[t_{k+1}, t_{k+1}+\tau\right)$. Rewrite (14) as

$$
\begin{aligned}
\dot{\varphi}(t) & =\alpha \varphi^{2}(t)+(\alpha \beta+\gamma) \varphi(t)+\beta \gamma \\
& =\alpha\left(\varphi^{2}(t)+\frac{\alpha \beta+\gamma}{\alpha} \varphi(t)+\frac{\beta \gamma}{\alpha}\right) \\
& =\alpha\left[\left(\varphi(t)+\frac{\alpha \beta+\gamma}{2 \alpha}\right)^{2}-\frac{(\gamma-\alpha \beta)^{2}}{4 \alpha^{2}}\right] .
\end{aligned}
$$

Denote $(\gamma-\alpha \beta)^{2} / 4 \alpha^{2}$ as $q^{2}$, and take the transform

$$
\varphi(t)+\frac{\alpha \beta+\gamma}{2 \alpha}=s(t), \quad d \varphi=d s,
$$

then

$$
\begin{aligned}
\tau & =\int_{t_{k+1}}^{t_{k+1}+\tau} d t=\frac{1}{\alpha} \int_{\varphi\left(t_{k+1}\right)}^{\varphi\left(t_{k+1}+\tau\right)} \frac{1}{\varphi^{2}+((\alpha \beta+\gamma) / \alpha) \varphi+\beta \gamma / \alpha} d \varphi \\
& =\frac{1}{\alpha} \int_{\varphi\left(t_{k+1}\right)+((\alpha \beta+\gamma) / 2 \alpha)}^{\varphi\left(t_{k+1}+\tau\right)+((\alpha \beta+\gamma) / 2 \alpha)} \frac{1}{s^{2}-q^{2}} d s \\
& =\left.\frac{1}{2 q \alpha} \ln \frac{s-q}{s+q}\right|_{\varphi\left(t_{k+1}\right)+((\alpha \beta+\gamma) / 2 \alpha)} \\
& =\frac{1}{\gamma-\alpha \beta} \ln \frac{\left(\varphi\left(t_{k+1}+\tau\right)+\beta\right)\left(\varphi\left(t_{k+1}\right)+\gamma / \alpha\right)}{\left(\varphi\left(t_{k+1}+\tau\right)+\gamma / \alpha\right)\left(\varphi\left(t_{k+1}\right)+\beta\right)} .
\end{aligned}
$$

The desired upper bound for $\sigma$ is obtained by solving the last equation in (19) with

$$
\varphi\left(t_{k+1}+\tau\right)=\sigma_{1}, \quad \varphi\left(t_{k+1}\right)=\sigma_{2},
$$

that is,

$$
\sigma_{2}=\frac{\gamma\left(\sigma_{1}+\beta\right)-\beta\left(\alpha \sigma_{1}+\gamma\right) e^{(\gamma-\alpha \beta) \tau}}{\left(\alpha \sigma_{1}+\gamma\right) e^{(\gamma-\alpha \beta) \tau}-\alpha\left(\sigma_{1}+\beta\right)} .
$$

The inequality $\sigma \leq \sigma_{2}<\sigma_{1}$ implies that (10) can be guaranteed for $t \in\left[t_{k+1}, t_{k+1}+\tau_{k+1}\right)$ by generating an event at time instant $t_{k+1}$.

In addition, another constraint needs to be enforced on $\sigma$ to guarantee that there is no event being generated for the time $t \in\left[t_{k+1}, t_{k+1}+\tau_{k+1}\right)$, that is, $\left\|e_{k+1}(t)\right\| /\|x(t)\|<\sigma$. At $t=t_{k+1}$, an event occurs, and the control error changes from $e_{k}(t)=K\left(x\left(t_{k}\right)-x(t)\right)$ to $e_{k+1}(t)=K\left(x\left(t_{k+1}\right)-x(t)\right)$. To avoid the out-of-order transmission for $t \in\left[t_{k+1}, t_{k+1}+\tau_{k+1}\right)$, the dynamic of $\left\|e_{k+1}(t)\right\| /\|x(t)\|$ should be bounded by $\sigma$. Follow the same arguments as (13) to get

$$
\begin{aligned}
\frac{d}{d t} \frac{\left\|e_{k+1}(t)\right\|}{\|x(t)\|}= & \frac{d}{d t} \frac{\left[e_{k+1}^{T}(t) e_{k+1}(t)\right]^{1 / 2}}{\left[x^{T}(t) x(t)\right]^{1 / 2}} \\
= & -\frac{e_{k+1}^{T}(t) K \dot{x}(t)}{\left\|e_{k+1}(t)\right\|\|x(t)\|}-\frac{x^{T}(t) \dot{x}(t)}{\|x(t)\|^{2}} \frac{\left\|e_{k+1}(t)\right\|}{\|x(t)\|} \\
\leq & \frac{\left\|e_{k+1}(t)\right\|\|K\|\|\dot{x}(t)\|}{\left\|e_{k+1}(t)\right\|\|x(t)\|} \\
& \left.+\frac{\|x(t)\|\|\dot{x}(t)\|\left\|e_{k+1}(t)\right\|}{\|x(t)\|\|x(t)\|} \frac{\|x(t)\|}{\|x(t)\|}\right) \frac{\|\dot{x}(t)\|}{\|x(t)\|} \\
= & \left(\|K\|+\frac{\left\|e_{k+1}(t)\right\|}{\| x(t)}\right. \\
\leq & \left(\|K\|+\frac{\left\|e_{k+1}(t)\right\|}{\|x(t)\|}\right) \\
& \times \frac{\|A+B K\|\|x(t)\|+\|B\|\left\|e_{k}(t)\right\|}{\|x(t)\|} \\
= & \left(\|K\|+\frac{\left\|e_{k+1}(t)\right\|}{\|x(t)\|}\right) \\
& \times\left(\|A+B K\|+\|B\| \frac{\left\|e_{k}(t)\right\|}{\|x(t)\|}\right),
\end{aligned}
$$

where $\left\|e_{k}(t)\right\| /\|x(t)\|$ is bounded by $\varphi(t)$, which can be found by solving the last equation in (19) with $\varphi\left(t_{k+1}\right)=$ $\sigma_{2}$. By the comparison principle, an upper bound for the evolution of the ratio $\left\|e_{k+1}(t)\right\| /\|x(t)\|$ can be immediately obtained by solving

$$
\dot{\phi}(t)=(\|K\|+\phi(t))(\|A+B K\|+\|B\| \varphi(t)),
$$

with $\left\|e_{k+1}(t)\right\| /\|x(t)\| \leq \phi(t)$. Furthermore, it follows from (23) and $\phi\left(t_{k+1}\right)=0$ that

$$
\begin{aligned}
\phi\left(t_{k+1}+\tau\right) \\
=\|K\|\left(\exp \left(\int_{t_{k+1}}^{t_{k+1}+\tau}(\|A+B K\|+\|B\| \varphi(t)) d t\right)-1\right) \\
\leq\|K\|\left(\exp \left(\int_{t_{k+1}}^{t_{k+1}+\tau}\left(\|A+B K\|+\|B\| \sigma_{1}\right) d t\right)-1\right) \\
=\|K\|\left(\exp \left(\left(\|A+B K\|+\|B\| \sigma_{1}\right) \tau\right)-1\right) \\
=\sigma_{3},
\end{aligned}
$$

which implies that there is no another event being triggered before the termination of the previous one if $\sigma \geq \sigma_{3}$. The lower bound of the difference between two consecutive event times is described by $\tau_{k}+\eta$, where $\eta$ is the time for $\varphi(t)$ to evolve from $\sigma_{3}$ to $\sigma$. Substituting the corresponding values in (19), thus

$$
\eta=\frac{1}{\gamma-\alpha \beta} \ln \frac{(\sigma+\beta)\left(\sigma_{3}+\gamma / \alpha\right)}{(\sigma+\gamma / \alpha)\left(\sigma_{3}+\beta\right)}
$$

is obtained. 
Hence, the following theorem can be concluded.

Theorem 3. For a given parameter $\tau$ and any $k \in \mathbb{N}$, the event condition in (4) with

$$
\sigma_{3} \leq \sigma \leq \sigma_{2}
$$

enforced for any $t \in\left[t_{k}, t_{k+1}\right)$ and the control law in (2) with $K$ given by (8) executed for any $t \in\left[t_{k}+\tau_{k}, t_{k+1}+\tau_{k+1}\right)$ guarantee that the system in (1) under the event-based control is asymptotically stable, and the inter-event intervals are lower bounded by $\tau_{k}+\eta$, where $\eta$ is given in (25).

Remark 4. Theorem 3 provides a useful way of design for both the feedback gain $K$ and the trigger parameter $\sigma$. Moreover, the information of the transmission delays is also involved. Therefore, the method can be used to tackle the case with time-varying network transmission delays. For given upper bound $\tau$ on the transmission delays, by solving (8), (21), (24), the corresponding feedback gain and trigger parameter can be obtained, which can be used to guarantee the required performance even though the transmission delays exist in the network communication.

Remark 5. The maximum $\tau$ can be solved by maximizing $\sigma_{1}$ in terms of (8) and letting $\sigma_{2}=\sigma_{3}$. However, how to find the optimal value of $\sigma_{1}$ is still open. Appropriately selecting the value of $\sigma_{1}$ will lead to a relatively larger value of the upper bound $\tau$.

Note that if $\tau_{k}=0$, that is, no transmission delay or the effect of the transmission delay can be omitted, Theorem 3 reduces to the result in the following corollary.

Corollary 6. For any $k \in \mathbb{N}$, the event condition in (4) with

$$
\sigma \leq \sigma_{1}
$$

enforced and the control law in (2) with $K$ given by (8) executed for any $t \in\left[t_{k}, t_{k+1}\right)$ guarantee that the system in (1) under the event-based control is asymptotically stable, and the inter-event intervals are lower bounded by $\eta$, where $\eta$ satisfies

$$
\eta=\frac{1}{\gamma-\alpha \beta} \ln \frac{(\sigma+\beta) \gamma}{(\alpha \sigma+\gamma) \beta} .
$$

Proof. The inequality $\sigma \leq \sigma_{1}$ implies that (10) can be guaranteed, thus the controller renders the closed-loop system asymptotically stable for any $t \in\left[t_{k}, t_{k+1}\right)$ and for any $k \in \mathbb{N}$. To estimate the inter-event time, the dynamic of $\left\|e_{k}(t)\right\| /\|x(t)\|$ should be bounded by $\varphi(t)$. Following the same arguments for $t \in\left[t_{k}, t_{k+1}\right)$, we have

$$
\eta=\frac{1}{\gamma-\alpha \beta} \ln \frac{\left(\varphi\left(t_{k}+\eta\right)+\beta\right)\left(\varphi\left(t_{k}\right)+\gamma / \alpha\right)}{\left(\varphi\left(t_{k}+\eta\right)+\gamma / \alpha\right)\left(\varphi\left(t_{k}\right)+\beta\right)} .
$$

Substituting the corresponding values $\varphi\left(t_{k}\right)$ and $\varphi\left(t_{k}+\eta\right)$ by 0 and $\sigma$, thus (28) is obtained.

Remark 7. The lower bound of the inter-event intervals provided in (28) is always positive if $\gamma \neq \alpha \beta$. It can be shown in the following. Without loss of generality, suppose $\gamma<\alpha \beta$, then

$$
\begin{gathered}
\gamma<\alpha \beta \Longleftrightarrow \sigma \gamma<\sigma \alpha \beta \Longleftrightarrow \sigma \gamma+\beta \gamma \\
<\sigma \alpha \beta+\beta \gamma \Longleftrightarrow \frac{(\sigma+\beta) \gamma}{(\sigma \alpha+\gamma) \beta}<1 .
\end{gathered}
$$

Thus both terms $\gamma-\alpha \beta$ and $\ln ((\sigma+\beta) \gamma /(\alpha \sigma+\gamma) \beta)$ are negative. Similarly, the positiveness can be proved for the case $\gamma>\alpha \beta$.

Remark 8. For the case $\gamma=\alpha \beta$, go back to (17), which can be written as

$$
\dot{\varphi}(t)=\alpha[\varphi(t)+\beta]^{2} .
$$

Take the transform

$$
\varphi(t)+\beta=s(t), \quad d \varphi=d s,
$$

then

$$
\begin{aligned}
\eta & =\int_{t_{k}}^{t_{k}+\eta} d t=\frac{1}{\alpha} \int_{\varphi\left(t_{k}\right)}^{\varphi\left(t_{k}+\eta\right)} \frac{1}{[\varphi+\beta]^{2}} d \varphi \\
& =\frac{1}{\alpha} \int_{\varphi\left(t_{k}\right)+\beta}^{\varphi\left(t_{k}+\eta\right)+\beta} \frac{1}{s^{2}} d s \\
& =-\left.\frac{1}{\alpha} \frac{1}{s}\right|_{\varphi\left(t_{k}\right)+\beta} ^{\varphi\left(t_{k}+\eta\right)+\beta} \\
& =\frac{1}{\alpha}\left[\frac{1}{\varphi\left(t_{k}\right)+\beta}-\frac{1}{\varphi\left(t_{k}+\eta\right)+\beta}\right] .
\end{aligned}
$$

The desired lower bound $\eta$ for the inter-event times is obtained when $\varphi\left(t_{k}\right)=0$ and $\varphi\left(t_{k}+\eta\right)=\sigma$, that is,

$$
\eta=\frac{\sigma}{\alpha \beta(\sigma+\beta)} .
$$

\section{Simulation Examples}

The event-based control strategy proposed in this paper is now applied to solve practical and numerical problems.

Example 9. Consider the unstable inverted pendulum system. This process, which was nonlinear, had been analyzed theoretically to obtain a linearized process model

$$
\dot{x}(t)=A x(t)+B u(t)
$$

with

$$
A=\left[\begin{array}{cccc}
0 & 1 & 0 & 0 \\
0 & 0 & -\frac{m g}{M} & 0 \\
0 & 0 & 0 & 1 \\
0 & 0 & \frac{g}{l} & 0
\end{array}\right], \quad B=\left[\begin{array}{c}
0 \\
\frac{1}{M} \\
0 \\
-\frac{1}{M l}
\end{array}\right],
$$

where $M=10$ is the cart mass, $m=1$ is the mass of the pendulum bob, $l=3$ is the length of the pendulum arm, and 
TABLE 1: The comparison in terms of inter-event intervals for several values of $\sigma$.

\begin{tabular}{lccccccc}
\hline Event condition parameter $\sigma$ & 0.01 & 0.02 & 0.03 & 0.04 & 0.05 & 0.06 & 0.07 \\
\hline The average inter-event interval & 0.0073 & 0.0123 & 0.0183 & 0.0234 & 0.0282 & 0.0338 & 0.0389 \\
The maximum inter-event interval & 0.6569 & 0.9188 & 1.4787 & 1.2648 & 1.0860 & 1.4672 & 1.3987 \\
The number of total events & 5466 & 3250 & 2183 & 1709 & 1417 & 1183 & 1028 \\
\hline
\end{tabular}

$g=10$ is the gravitational acceleration. The states $x(t)=$ $\left[x_{1}(t), x_{2}(t), x_{3}(t), x_{4}(t)\right]^{T}$ are the cart position and velocity, the pendulum angular position and velocity, respectively. The initial state of the system is chosen as $x_{0}=\left[\begin{array}{llll}0.98 & 0 & 0.2 & 0\end{array}\right]^{T}$. Since the eigenvalues of the matrix $A$ are $-\sqrt{g / l}, 0,0, \sqrt{g / l}$, the open-loop system is unstable. The following feedback gain

$$
K=\left[\begin{array}{llll}
2 & 12 & 378 & 210
\end{array}\right]
$$

is chosen in the design of the event-triggered scheme.

Consider the case when $\tau_{k}=0$. Applying Corollary 6 with feedback gain in (37) and $Q=I$, the corresponding $P$ is given by

$$
P=10^{3} \times\left[\begin{array}{llll}
0.0046 & 0.0089 & 0.0589 & 0.0343 \\
0.0089 & 0.0251 & 0.1694 & 0.0988 \\
0.0589 & 0.1694 & 1.1536 & 0.6726 \\
0.0343 & 0.0988 & 0.6726 & 0.3926
\end{array}\right]
$$

The value of $\sigma_{1}$ is solved by (11) as 0.0781 . It can be concluded that system stability is guaranteed for any parameter $\sigma \leq 0.0781$. In this case, taking $\sigma=0.0781$, and using the event triggering condition in (4), a simulation is conducted for $t \in[0,40]$. It can be calculated that the event-based control algorithm in this paper leads to a maximum interevent interval 1.0414, and the average inter-event time is 0.0428 . Comparing with the average inter-event interval less than $10^{-5}$ in [21], the improvement over the result in [21] on the average inter-event time is obvious. Under the same conditions, the event instants and the inter-event intervals are shown in Figure 2, and the state responses of the system in (3) are shown in Figure 3. In addition, the comparison for different values of $\sigma$ chosen from the feasible range is reported in Table 1. From Table 1, it can be found that increasing the value of $\sigma$ has the effect of increasing the average inter-event interval but not the maximum interevent interval. The intuition behind the statement is that the ratio between the norms of the control error and the state will need more time to reach the threshold as the value of $\sigma$ increases.

Example 10. Consider a second-order linear control system described by

$$
\left[\begin{array}{c}
\dot{x}_{1}(t) \\
\dot{x}_{2}(t)
\end{array}\right]=\left[\begin{array}{cc}
0 & 1 \\
-2 & 3
\end{array}\right]\left[\begin{array}{l}
x_{1}(t) \\
x_{2}(t)
\end{array}\right]+\left[\begin{array}{l}
0 \\
1
\end{array}\right] u(t),
$$

and the controller

$$
u(t)=x_{1}\left(t_{k}\right)-4 x_{2}\left(t_{k}\right)
$$

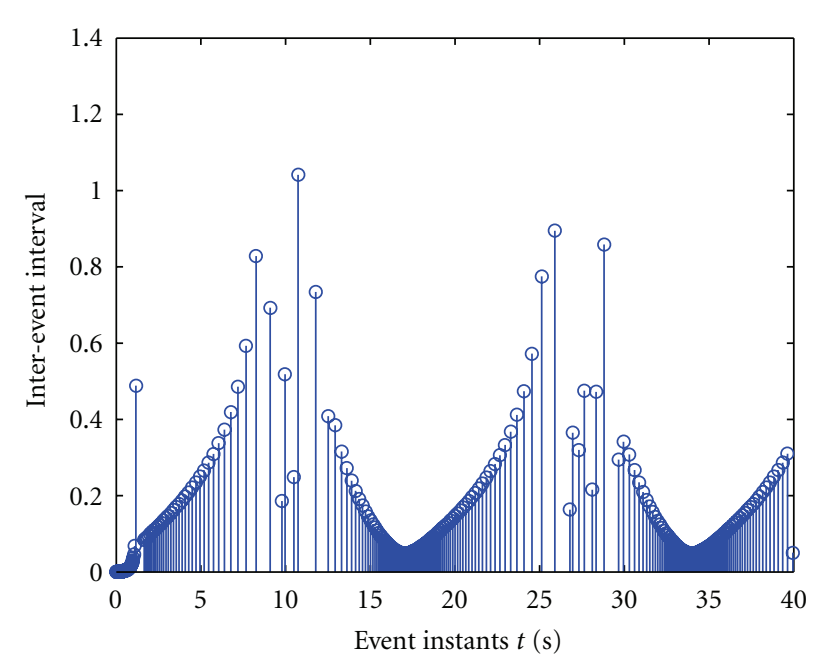

Figure 2: The event instants and inter-event intervals with event triggering condition in (4).

is designed to stabilize the closed-loop system. Choose

$$
Q=\left[\begin{array}{ll}
\frac{1}{2} & \frac{1}{4} \\
\frac{1}{4} & \frac{1}{2}
\end{array}\right],
$$

then $P$ is obtained via solving the Lyapunov equation:

$$
P=\left[\begin{array}{cc}
1 & \frac{1}{4} \\
\frac{1}{4} & 1
\end{array}\right] .
$$

Using $\sigma_{2}=\sigma_{3}$ with $\alpha=1, \beta=4.1231, \gamma=1.618$, and $\sigma_{1}=0.2139$, the upper bound for the random delays $\tau_{k}$ is computed as 0.014 , that is, the closed-loop system can tolerate the transmission delays bounded by 0.014 . For $\tau=0.005 \mathrm{~s}$ and according to Theorem 3 , any $\sigma$ satisfying $0.0379 \leq \sigma \leq 0.1748$ can be selected. The theoretical value for the minimum inter-event interval corresponding to $\sigma=0.1064$ is 0.0097 . The evolution of the Lyapunov function $V(t)$ based on the event condition in (4) is depicted in Figure 4. From the simulation result, it can be seen that the event-based control system is robust to time-varying transmission delays. Figure 5 shows the evolution of $\|e(t)\|$ based on the event condition in (4) in the presence of time delays. In this figure, an event is generated when the control error norm reaches the dash-dot line, and the control signal is transmitted to the actuator node via the network. Therefore, the error will never go beyond the dash line which 


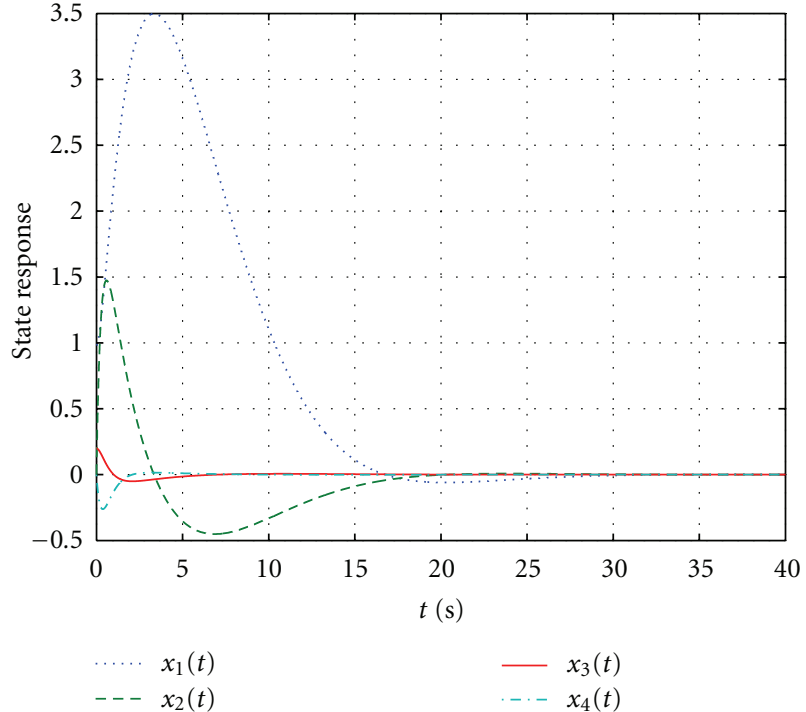

Figure 3: The state responses of the system in (3) with event triggering condition in (4).

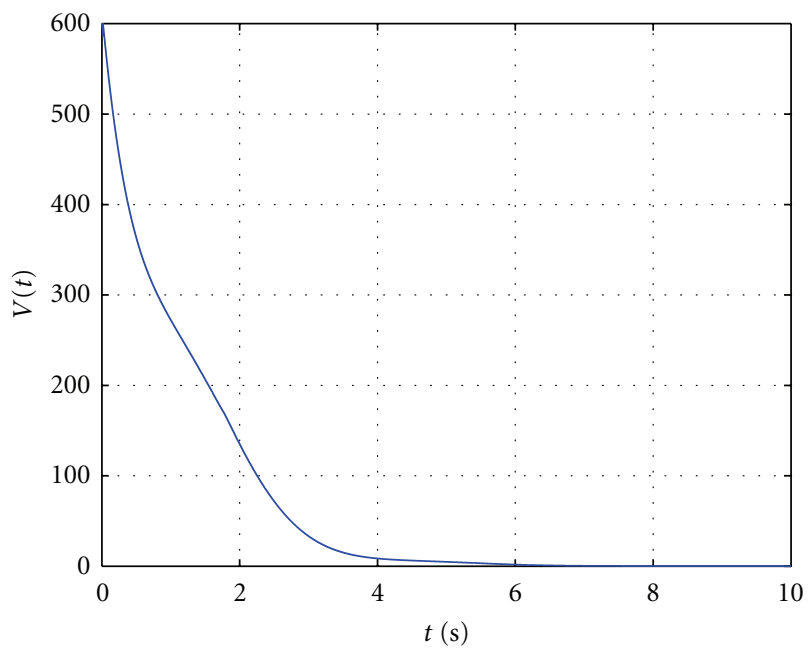

Figure 4: Evolution of $V(t)$ for $\sigma=0.1064$ and initial condition $\left(x_{1}(0), x_{2}(0)\right)=(10,20)$.

guarantees the asymptotic stability. The comparison between the estimated and the simulated evolution of $\|e(t)\| /\|x(t)\|$ is provided over one of the shortest inter-event intervals $[0.1468,0.1625]$ in Figure 6 . The gap between the simulated and the estimated is relatively small. Thus, the equation developed in (25) guarantees a tight lower bound of the interevent intervals.

\section{Conclusions}

To save communication bandwidth, a new event-triggered communication strategy has been developed for control over networks, which can be used to determine when the control signals will be transmitted. The event detector is based on the control error; the control is implemented via

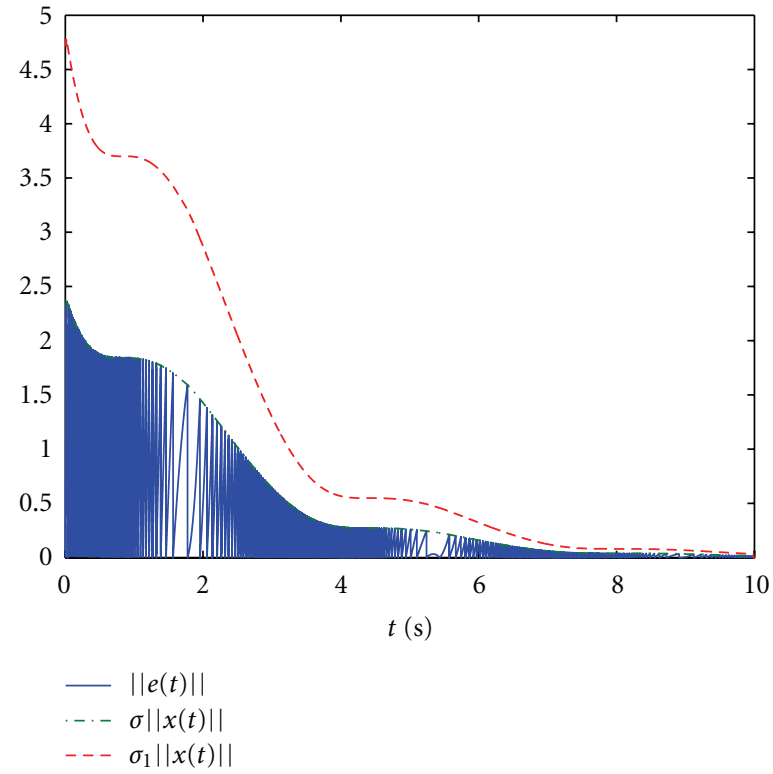

Figure 5: Evolution of $\|e\|, \sigma\|x\|, \sigma_{1}\|x\|$.

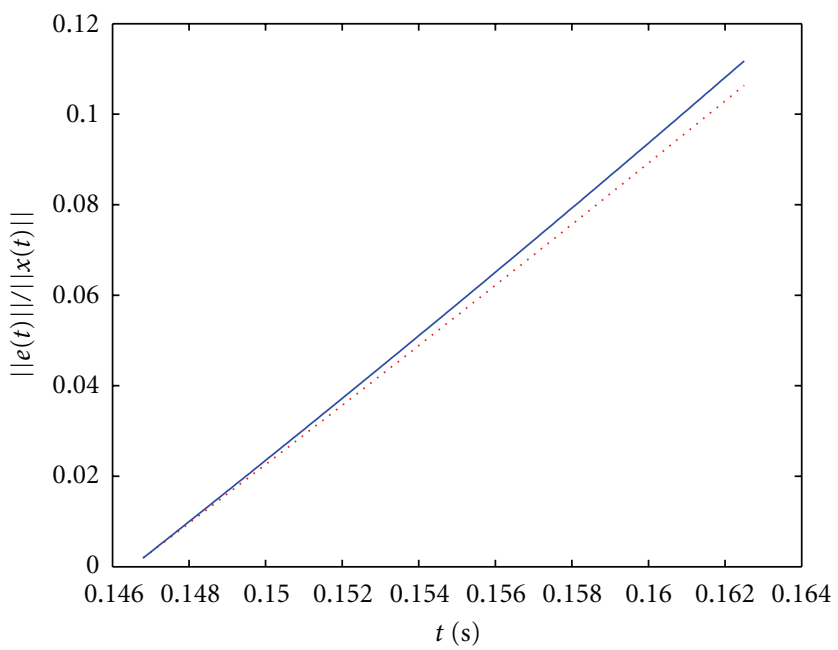

Simulated ||$e(t)|| /|| x(t) \mid$

- Theoretical estimate for $\|e(t)\| /|| x(t) \|$

FIGURE 6: Evolution of $\|e(t)\| /\|x(t)\|$ obtained by simulation and its estimation according to (19) for $\sigma=0.1064$.

sample and hold devices. Notice that the results consider the effect of the random time-varying network-induced delays. Two examples detail the advantages of event-based implementation. However, this approach has some limitations. It can be seen that the constructed event detector requires delicate hardware to monitor the control signal and test the logic condition continuously. To overcome this disadvantage, the strategy of discrete detection will be proposed in our future work, where the event detector only needs a supervision of the event condition at discrete sampling instants. Moreover, the parameter of the event 
detector is chosen with the assumption that the controller gain is designed to guarantee the global asymptotic stability of the closed-loop system in advance without considering the effect of network transmission delays. The method of jointly designing the parameter of the event detector and the controller gain will also be considered in our future work to give higher resource utilization and better performance.

\section{Acknowledgment}

This work was supported by NSERC and an iCORE Ph.D. Recruitment Scholarship from the Province of Alberta.

\section{References}

[1] J. P. Hespanha, P. Naghshtabrizi, and Y. Xu, "A survey of recent results in networked control systems," Proceedings of the IEEE, vol. 95, no. 1, pp. 138-162, 2007.

[2] L. A. Montestruque and P. Antsaklis, "Stability of model-based networked control systems with time-varying transmission times," IEEE Transactions on Automatic Control, vol. 49, no. 9, pp. 1562-1572, 2004.

[3] L. Zhang, Y. Shi, T. Chen, and B. Huang, "A new method for stabilization of networked control systems with random delays," IEEE Transactions on Automatic Control, vol. 50, no. 8, pp. 1177-1181, 2005.

[4] L. Bakule and M. de la Sen, "Non-Fragile controllers for a class of time-delay nonlinear systems," Kybernetika, vol. 45, no. 1, pp. 15-32, 2009.

[5] L. Bakule and M. de la Sen, "Decentralized resilient $H_{\infty}$ observer-based control for a class of uncertain interconnected networked systems," in Proceedings of the American Control Conference (ACC '10), pp. 1338-1343, Baltimore, Md, USA, 2010.

[6] E. Kofman and J. H. Braslavsky, "Level crossing sampling in feedback stabilization under data-rate constraints," in Proceedings of the 45th IEEE Conference on Decision and Control, (CDC '06), pp. 4423-4428, December 2006.

[7] K. J. Åström and B. M. Bernhardsson, "Comparison of Riemann and Lebesgue sampling for first order stochastic systems," in Proceedings of the 41st IEEE Conference on Decision and Control, pp. 2011-2016, December 2002.

[8] M. Miskowicz, "Send-on-delta concept: an event-based data reporting strategy," Sensors, vol. 6, no. 1, pp. 49-63, 2006.

[9] P. G. Otanez, J. R. Moyne, and D. M. Tilbury, "Using deadbands to reduce communication in networked control systems," in Proceedings of the American Control Conference, pp. 3015-3020, Anchorage, Alaska, USA, May 2002.

[10] P. Tabuada, "Event-triggered real-time scheduling of stabilizing control tasks," IEEE Transactions on Automatic Control, vol. 52, no. 9, pp. 1680-1685, 2007.

[11] W. P. M. H. Heemels, J. H. Sandee, and P. P. J. Van Den Bosch, "Analysis of event-driven controllers for linear systems," International Journal of Control, vol. 81, no. 4, pp. 571590, 2008.

[12] M. Velasco, P. Martí, and E. Bini, "Control-driven tasks: modeling and analysis," in Proceedings of the Real-Time Systems Symposium, pp. 280-290, Barcelona, Spain, 2008.

[13] M. Mazo and P. Tabuada, "On event-triggered and selftriggered control over sensor/actuator networks," in Proceedings of the 47th IEEE Conference on Decision and Control, (CDC '08), pp. 435-440, Cancun, Mexico, December 2008.
[14] J. Lunze and D. Lehmann, "A state-feedback approach to event-based control," Automatica, vol. 46, no. 1, pp. 211-215, 2010.

[15] D. V. Dimarogonas and K. H. Johansson, "Event-triggered cooperative control," in Proceedings of the European Control Conference, pp. 3015-3020, Budapest, Hungary, 2009.

[16] X. Wang and M. D. Lemmon, "Event-triggering in distributed networked systems with data dropouts and delays," in Hybrid Systems: Computation and Control, R. Majumdar and P. Tabuada, Eds., vol. 5469 of Lecture Notes in Computer Science, pp. 366-380, Springer, Berlin, Germany, 2009.

[17] X. Wang and M. Lemmon, "On event design in event-triggered feedback systems," Automatica, vol. 47, no. 10, pp. 2319-2322, 2011.

[18] D. Yue, E. Tian, and Q. Han, "A delay system method to design of event-triggered control of networked control systems," in Proceedings of the 50th IEEE Conference on Decision and Control and European Control Conference, pp. 1668-1673, Orlando, Fla, USA, 2011.

[19] J. Sijs and M. Lazar, "On event based state estimation," in Hybrid Systems: Computation and Control, R. Majumdar and P. Tabuada, Eds., vol. 5469 of Lecture Notes in Computer Science, pp. 336-350, Springer, Berlin, Germany, 2009.

[20] H. K. Khalil, Nonlinear Systems, Prentice Hall, 3rd edition, 2002.

[21] P. Tabuada and X. Wang, "Preliminary results on statetrigered scheduling of stabilizing control tasks," in 45th IEEE Conference on Decision and Control 2006, CDC, pp. 282-287, San Diego, Calif, USA, December 2006. 

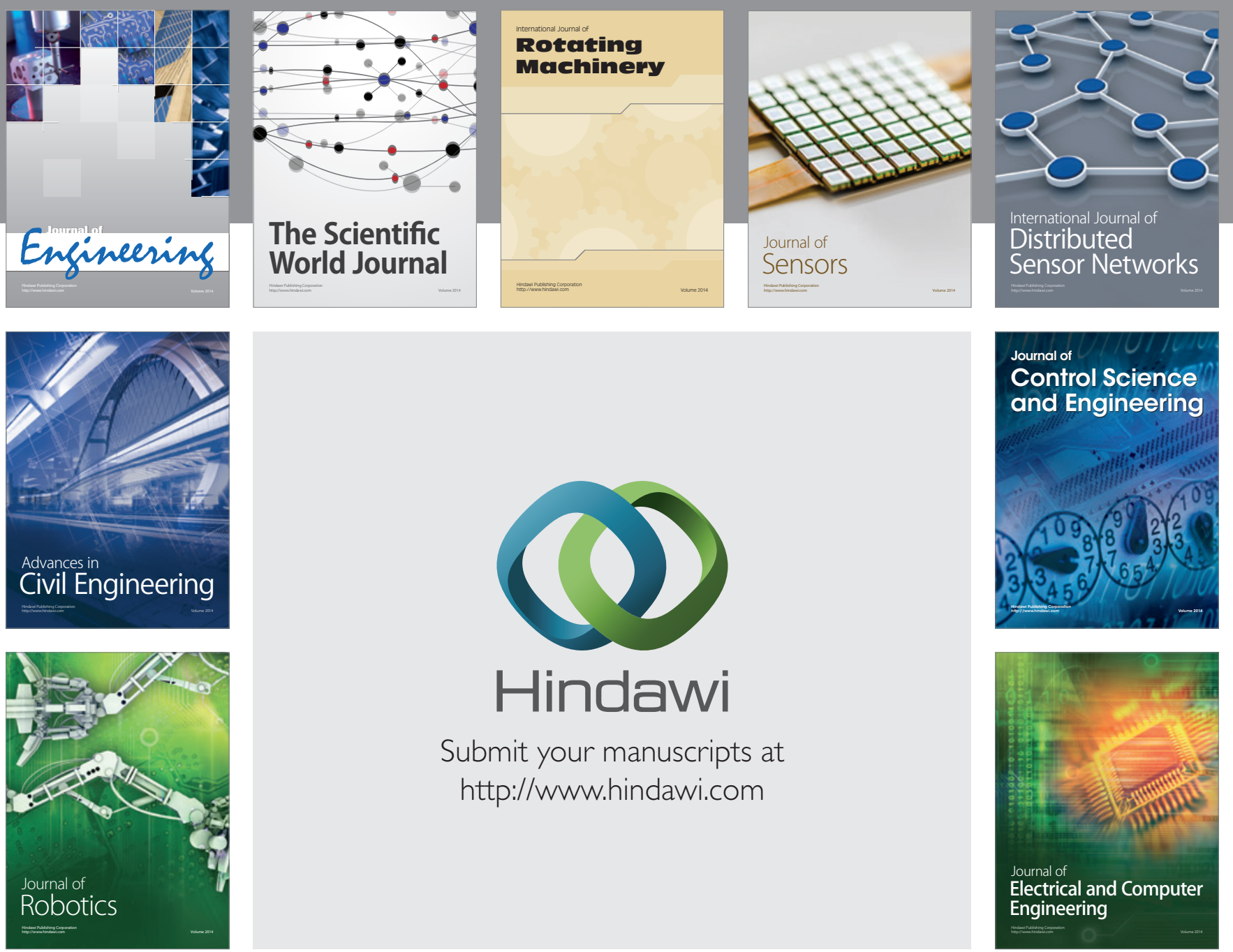

Submit your manuscripts at

http://www.hindawi.com
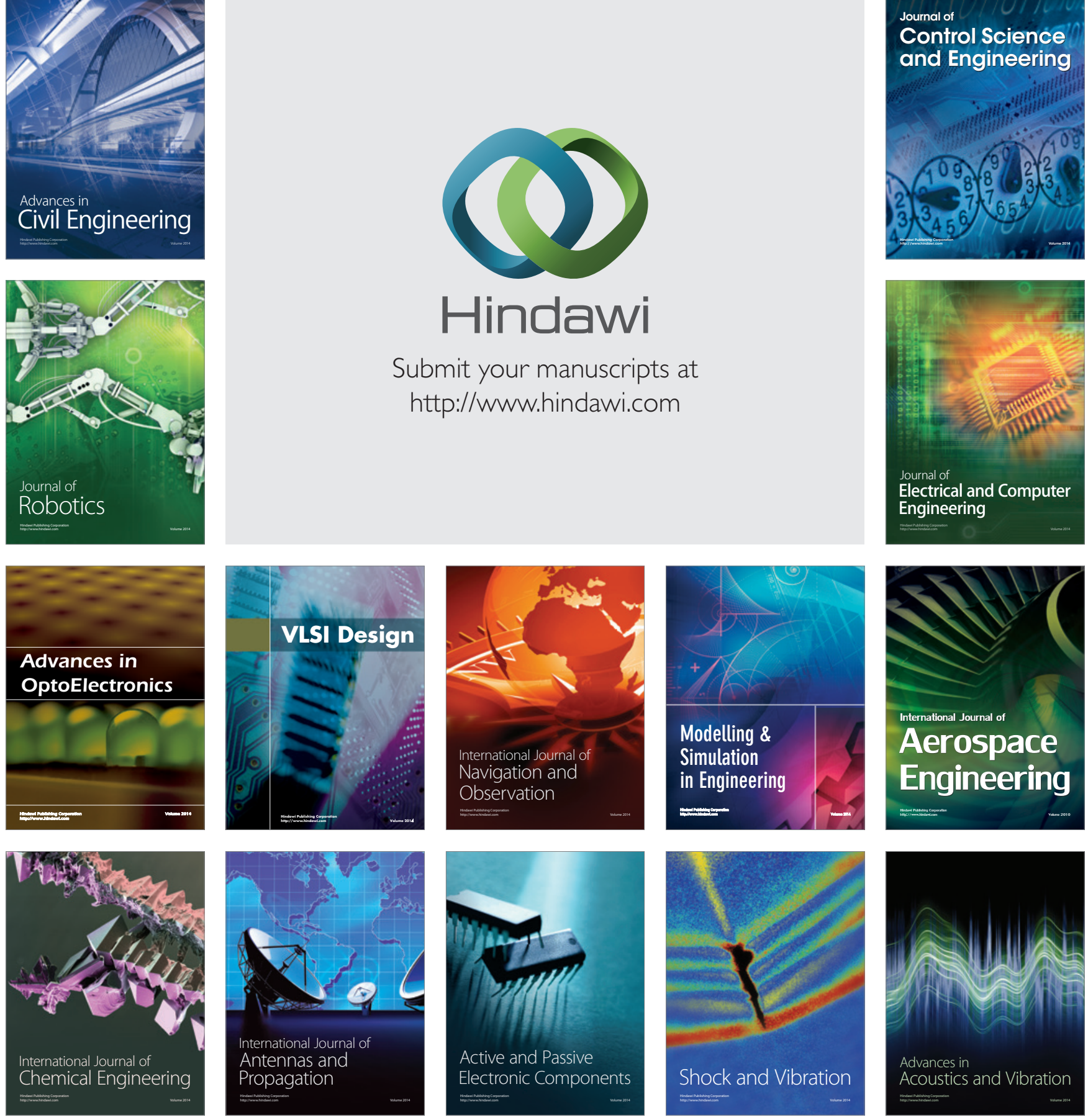